\title{
Déterminants clés de la pratique optimale de l'allaitement exclusif au niveau de la communauté urbaine de Niamey, Niger
}

\author{
Halima DIADIE OUMAROU ${ }^{1 *}$ et Abdourahamane BALLA ${ }^{2}$ \\ I'Département des Productions Végétales/Faculté d'Agronomie/Université Abdou Moumouni, Niamey-Niger. \\ ${ }^{2}$ CRESA / Faculté d'Agronomie/ Université Abdou Moumouni, Niamey-Niger. \\ *Auteur correspondant; E-mail : dialima75@yahoo.fr
}

\begin{tabular}{lll}
\hline Received: 17-09-2020 & Accepted: 29-05-2021 & Published: 30-06-2021 \\
\hline
\end{tabular}

\section{RESUME}

L'allaitement maternel exclusif (AME), mode d'alimentation de prédilection pour les nourrissons, demeure insuffisamment pratiqué. L'objectif général de cette étude était de contribuer à la pratique optimale de l'AME au niveau de la communauté urbaine de Niamey. Cette étude transversale, d'une durée de 3 mois a porté sur un échantillon de 400 mères d'enfants de 0-6 mois choisi, par commodité au cours de leurs consultations nourrisson au niveau des centres de santé. Les résultats ont montré que l'initiation précoce à l'allaitement maternel a concerné $48 \%$ d'enfants ayant été mis au sein dans l'heure qui suit la naissance, et que seulement 33, $5 \%$ des mères ont pratiqué l'AME. Cependant, l'analyse statistique traduit une association entre l'allaitement maternel exclusif et certains des facteurs testés. Ces facteurs clés permettant d'optimiser la pratique de l'AME demeurent: la connaissance de la durée et de la définition de l'AME, la connaissance des avantages de l'AME pour la mère tels que : retarde la fertilité et renforce le lien mère/enfant ; la connaissance des avantages pour l'enfant notamment: enfant grossit bien ou l'enfant est toujours en bonne santé et enfin le caractère économique de l'AME. Ces données permettent une meilleure orientation des interventions axées sur la pratique optimale de l'allaitement maternel à Niamey.

(C) 2021 International Formulae Group. All rights reserved.

Mots clés : Déterminants clés, allaitement maternel exclusif, pratique optimale, nourrissons, Niamey.

\section{Key determinants of exclusive breastfeeding optimal practice in the urban community of Niamey, Niger}

\begin{abstract}
Exclusive breastfeeding, the preferred feeding mode for infants, remains insufficiently practised. The overall objective of this study was to contribute to the optimal practice of exclusive breastfeeding in Niamey urban community. This cross-sectional study included a sample of 400 mothers of children aged 0-6 months chosen for convenience during their infant consultations at the health centre level. The results showed that $48 \%$ of children who were breast-fed within one hour of birth were introduced early to breastfeeding, and only $33.5 \%$ of mothers practiced exclusive breastfeeding. However, the statistical analysis shows an association between exclusive breastfeeding and some of the factors tested. These key factors for optimizing the practice of exclusive breastfeeding remain: knowledge of the duration and definition of breastfeeding, knowledge of the benefits of
\end{abstract}


breastfeeding for the mother such as: delays fertility and strengthens the mother/child bond; knowledge of the benefits for the child in particular: child grows well or the child is always healthy and finally the economic character of the breastfeeding. These data allow a better orientation of interventions focused on the optimal practice of breastfeeding in Niamey.

(C) 2021 International Formulae Group. All rights reserved.

Keywords: Key determinants, exclusive breastfeeding, optimal practice, infants, Niamey.

\section{INTRODUCTION}

Une mauvaise alimentation, surtout durant les 1000 jours d'un enfant, peut avoir des conséquences irréversibles sur sa santé. Aussi, pour des millions d'enfants, cela implique un retard de croissance qui les affectera pour le reste de leur vie (UNICEF, 2013). En effet, il faut noter une prévalence de $47,8 \%$ et $15 \%$ respectivement pour le retard de croissance et l'émaciation enregistré en 2018 chez les enfants de moins de 59 mois au Niger (INS, 2018). Ainsi, l'allaitement maternel exclusif malgré son efficacité et son caractère très économique, demeure, pendant les six premiers mois du bébé, une pratique insuffisamment pratiquée dans certaines régions du globe. En effet, seuls deux enfants âgés de moins de 6 mois sur cinq bénéficient encore de l'allaitement exclusif recommandé (Belamy, 2004). Aussi, un tiers des décès des enfants de moins de 5 ans dans le monde est dû à la sous-nutrition. Celle-ci expose, en effet, l'enfant à un risque beaucoup plus élevé de succomber ou d'être gravement touché par les maladies infantiles. Par ailleurs, durant les six premiers mois, le nourrisson non allaité présente un risque de décès 14 fois plus élevé (toutes causes confondues) que le nourrisson exclusivement nourri au sein (UNICEF, 2013). L'allaitement maternel constitue, en effet le premier «vaccin» du nourrisson. Il contribue à le protéger contre la diarrhée, les infections respiratoires aiguës et d'autres problèmes de santé. Au Niger, le taux d'allaitement maternel exclusif présente, entre 2006 et 2012; une faible amélioration qui est passée de $14 \%$ à 23\% (MSP, 2017). Depuis, ce taux évolue positivement mais demeure assez faible de nos jours. Ainsi, il est impératif de caractériser les déterminants clés pour une pratique optimale de l'allaitement maternel exclusif au Niger. La présente étude s'inscrit dans ce même ordre d'idée et contribue à l'amélioration de la pratique de l'allaitement exclusif au niveau de la communauté urbaine de Niamey.

\section{MATERIEL ET METHODES \\ Matériel \\ Présentation des sites d'étude}

L'étude a été réalisée au niveau de 4 centres de santé intégrés (CSI) de la communauté urbaine de Niamey. En effet, les CSI concernés sont : le CSI Abidjan, le CSI koiratégui et le CSI lazaret ainsi que le CSI Gaweye, situés respectivement dans le $2^{\text {ème }}$ arrondissement et le 5ème arrondissement de Niamey. Ces CSI sont tous de type 2 c'est-à-dire dotés d'une maternité. Ils constituent la structure sanitaire de premier recours pour la population.

\section{Population d'étude}

La population d'étude est composée des mères d'enfants âgés de 0 -6mois venues en consultation nourrisson au moment de l'enquête.

\section{Critères d'inclusion}

Toutes les mères dont les enfants ont l'âge compris entre 0 à 6 mois venues en consultation nourrisson ayant accepté de répondre aux questions.

\section{Critères de non inclusion}

Toutes les mères d'enfants âgés de 0 à 6 mois venues en consultations des nourrissons qui ne sont pas en état de répondre aux questions et/ ou les mères qui ont abandonnée l'entretien en cours.

\section{Outils de collecte de données}

L'outil de collecte des données est un questionnaire adressé aux mères d'enfants de 0 à 6 mois:

- caractéristiques sociodémographiques : le niveau d'instruction, l'activité principale, l'âge ainsi que le statut matrimonial 
- connaissance des mères sur l'alimentation du nourrisson de 0-6 mois en général et en AME en particulier, pratique de AME, avantages de AME: l'initiation à l'allaitement, la prise de colostrum, la pratique de l'allaitement, l'introduction d'aliment de complément.

\section{Outils de traitement de données}

Il s'agit principalement du logiciel SPSS 23 et le logiciel Excel version 2010 ayant servis respectivement à l'analyse statistique et à l'élaboration des figures.

\section{Méthodes d'étude \\ Type d'étude}

Il s'agit d'une étude transversale visant à une meilleure compréhension de la pratique optimale de l'allaitement maternel exclusif au niveau de Niamey.

\section{Echantillonnage}

La taille de l'échantillon a été déterminée par sélection aléatoire des mères au niveau des 4 sites d'étude. En effet, les mères ont été questionnées au fur et à mesure qu'elles se présentaient en consultation nourrisson ou à une séance de vaccination. Ainsi, la taille finale est de 400 mères d'enfants de 0 à 6 mois.

\section{Collecte des données}

La collecte des données s'est déroulée sur une période de deux mois, allant du 17 juin au 17 Août 2019. Les données ont été collectées à travers un entretien semi structuré.

\section{Traitement et analyse des données}

Les données collectées ont été saisies et analysées à l'aide du logiciel SPSS version 23. Les tableaux et les figures ont été confectionnés à l'aide du tableur Excel et la rédaction du mémoire a été faite sur Microsoft Word 2016. Le test khi-deux de Pearson a été utilisé au seuil 5\% pour tester la relation significative entre la pratique de l'AME et les facteurs/déterminants qui l'associent.

\section{Considérations éthiques et administratives éventuelles}

L'autorisation de mener l'enquête a été obtenue auprès des responsables de la DRSP, des responsables des districts Niamey II et V. Les mères enquêtées ont été informées sur le but de l'enquête et sur la manière de procéder. Le consentement éclairé des enquêtées a été demandé et des dispositions ont été prises pour éviter tout incident pouvant survenir au cours de l'entretien. La confidentialité et l'anonymat des données ont été garantis.

\section{RESULTATS \\ Caractérisation socio-économiques des mères}

Les résultats du Tableau 1 révèlent que les enfants de 0 à 6 mois ciblés sont issus de mères relativement jeunes puisque les tranches d'âge dominantes sont $15-24$ ans et $25-34$ ans avec respectivement $40,3 \%$ et $47,5 \%$. Aussi, ces mères en majorité, sont scolarisées et représentent près de $81,5 \%$. Cependant, la plupart d'entre elles sont des ménagères $(57,4 \%)$, tandis que le reste demeure des fonctionnaires $(8,8 \%)$, commerçantes $(16 \%)$, couturières $(2,8 \%)$ ou bien de élèves/étudiantes (15\%). Il convient de relever que ces enfants de 0 à 6 mois proviennent d'une part à $74 \%$ de mères ayant entre 1 à 3 enfants de moins de 59 mois en plus. D'autre part, ces enfants de 0 et 6 mois ont entre 4 à 6 frères de moins de 59 mois $(26 \%)$.

\section{Initiation à l'allaitement maternel}

Les résultats du Tableau 2 font ressortir une proportion moyenne de mères $(48 \%)$ qui ont précocement mis leurs enfants au sein. Aussi, certaines mères ont avancé avoir initié leurs enfants à l'allaitement dans les 24 heures suivant la naissance (39\%). Cependant, $11 \%$ de mères ont attendu 48 heures voire 72 heures avant de mettre l'enfant au sein pour la première fois. Parallèlement, il faut noter $98 \%$ de ces enfants ont pu bénéficier du colostrum.

\section{Connaissances en matière d'allaitement maternel exclusif chez les mères}

Les connaissances des mères en matière d'allaitement maternel exclusif a été testées et les résultats consignés dans le Tableau 3 révèlent une relation statistiquement significative, $\mathrm{P}$-value $=0,000$ pour les variables définition et la durée l'AME donnés par les mères. Aussi, concernant les avantages de l'AME pour la mère, seuls l'espacement des grossesses et l'entretien de la relation mère- 
enfant sont statistiquement significatifs avec une P-value respective de 0,005 et 0,000. Quant aux avantages de l'AME pour l'enfant, seules les variables «l'enfant grossi bien » et « la diminution de maladies chez l'enfant » ont une association significative avec une $\mathrm{P}$-value de 0,000. Enfin, l'intérêt économique rapporté par les mères est aussi statistiquement significatif à la pratique de l'AME.

\section{Pratique d'allaitement maternel exclusif et facteurs associés \\ Pratique de l'allaitement maternel exclusif}

L'analyse de la Figure 1 montre qu'une proportion encore faible de mères ayant pratiqué l'allaitement exclusif. En effet, 33,5\% d'enfants ont pu bénéficier de l'allaitement exclusif contre $66,5 \%$ d'enfants n'ayant pas eu cette chance.

Déterminants sociodémographiques de la pratique optimale d'allaitement maternel exclusif

Les variables sociodémographiques rapportées à la variable pratique de l'allaitement maternel exclusif démontrent une association non significative dans l'ensemble (Tableau 4). En effet, la relation entre l'âge de la mère et la pratique de l'AME n'est pas significative puisque la $\mathrm{P}$-value est de 0,524.
Néanmoins, il faut noter que les mères d'âge compris entre 25 et 34 ans pratiquent beaucoup plus l'AME $(50,8 \%)$ que les mères des tranches d'âge : $15-24$ ans et 35 - plus. Ce constat est valable pour le niveau d'instruction des mères qui n'influence pas la pratique de l'AME comme le démontre la P-value de 0,821 . Aussi, il convient de noter que les variables profession, milieu de vie et parité n'ont aucune association significative avec cette pratique d'AME avec ces P-value respectives : 0,251; 0,953 et 0,152 . Par ailleurs, la tendance de la pratique de l'AME intra variables montre un pourcentage assez élevé de mères instruites et une proportion élevée de multipares et de ménagères qui ont allaité exclusivement leurs enfants.

\section{Allaitement maternel exclusif et introduction d'aliments de complément}

La Figure 2 traite des aliments qui sont donnés aux nourrissons à la naissance. Ainsi, l'eau bénite a été donnée à $53,39 \%$ d'enfants suivie par le jus de datte avec $36,44 \%$. L'eau simple et le miel ont également été donnés respectivement à 5,93\% et $4,23 \%$ d'enfants de cette zone d'étude. Cette introduction de ces aliments a limité l'initiation à l'allaitement et ou la pratique de l'allaitement exclusif.

Tableau 1 : Caractéristiques sociodémographiques des mères.

\begin{tabular}{lcc}
\hline Caractéristiques & \multicolumn{2}{c}{ Zone d'étude } \\
\cline { 2 - 3 } & Effectif & Pourcentage (\%) \\
\hline Tranche d'âge de la mère & $\mathbf{1 6 1}$ & $\mathbf{4 0 , 3}$ \\
15 - 24 ans & $\mathbf{1 9 0}$ & $\mathbf{4 7 , 5}$ \\
$25-34$ ans & $\mathbf{4 9}$ & $\mathbf{1 2 , 2}$ \\
35 ans et Plus & & \\
\hline Sexe de l'enfant & $\mathbf{2 0 4}$ & $\mathbf{5 1}$ \\
Masculin & $\mathbf{1 9 6}$ & $\mathbf{4 9}$ \\
Féminin & & \\
Niveau d'instruction de la mère & $\mathbf{6 4}$ & $\mathbf{1 6}$ \\
Coranique & $\mathbf{7 4}$ & $\mathbf{1 8 , 5}$ \\
Analphabète & $\mathbf{9 7}$ & $\mathbf{2 4 , 3}$ \\
Primaire & $\mathbf{1 1 7}$ & $\mathbf{2 9 , 2}$ \\
Secondaire & $\mathbf{4 8}$ & $\mathbf{1 2}$ \\
Supérieur & & $\mathbf{9 9 , 7}$ \\
\hline Situation matrimoniale & $\mathbf{3 9 9}$ & $\mathbf{0 , 3}$ \\
Mariée & $\mathbf{1}$ & \\
Célibataire & & \\
\hline
\end{tabular}




\begin{tabular}{lcc}
\hline Profession de la mère & $\mathbf{6 0}$ & $\mathbf{1 5}$ \\
Elève/étudiante & $\mathbf{6 4}$ & $\mathbf{1 6}$ \\
Commerçante & $\mathbf{3 5}$ & $\mathbf{8 , 8}$ \\
Fonctionnaire & $\mathbf{2 3 0}$ & $\mathbf{5 7 , 4}$ \\
Ménagère & $\mathbf{1 1}$ & $\mathbf{2 , 8}$ \\
Couturière & $\mathbf{2 9 6}$ & $\mathbf{7 4}$ \\
\hline Nombre enfants de 0 à 59 mois & $\mathbf{1 0 4}$ & $\mathbf{2 6}$ \\
1 à 3 & & \\
4 à 6 & $\mathbf{2 2 3}$ & $\mathbf{5 5 , 7 5}$ \\
\hline Milieu de résidence & $\mathbf{1 7 7}$ & $\mathbf{4 4 , 2 5}$ \\
Centre-ville & & \\
Périphérie &
\end{tabular}

Tableau 2 : Initiation à l'allaitement et prise de colostrum dans la zone d'étude.

\begin{tabular}{lcc}
\hline & \multicolumn{2}{c}{ Prise de colostrum } \\
\cline { 2 - 3 } Période d'Initiation à l'allaitement & Oui $(\boldsymbol{\%})$ & Non $(\boldsymbol{\%})$ \\
\hline Dans l'heure de naissance & $\mathbf{4 8}$ & $\mathbf{1 , 5}$ \\
\hline Dans les 24 heures de naissance & $\mathbf{3 9}$ & $\mathbf{0}$ \\
\hline Après 24 heures de naissance & $\mathbf{1 1}$ & $\mathbf{0 , 5}$ \\
\hline Total & $\mathbf{9 8}$ & $\mathbf{2}$ \\
\hline
\end{tabular}

Tableau 3: Facteurs de connaissance influençant la pratique optimale de l'allaitement maternel exclusif.

\begin{tabular}{|c|c|c|}
\hline Variables & Proportion (\%) & P-value \\
\hline Définition de l'AME & & $\mathbf{0 , 0 0 0}$ \\
\hline Lait maternel + eau & 3 & \\
\hline Lait maternel seul & 97 & \\
\hline Durée de l'AME & & $\mathbf{0 , 0 0 0}$ \\
\hline 0 - 3 mois & $\mathbf{0}$ & \\
\hline $0-4$ mois & $\mathbf{0}$ & \\
\hline $0-5$ mois & 1,5 & \\
\hline $0-6$ mois & 98,5 & \\
\hline \multicolumn{3}{|l|}{ Avantages de l'AME pour la mère } \\
\hline Espacement des grossesses & 11,9 & 0,005 \\
\hline Perte de poids & 1,5 & 0,158 \\
\hline Réduit le risque de cancer & 4,5 & 0,385 \\
\hline Entretien de la relation mère - enfant & 92,5 & 0,000 \\
\hline \multicolumn{3}{|l|}{ Avantages de l'AME pour l'enfant } \\
\hline Enfant devient intelligent & 10,9 & 0,118 \\
\hline Enfant grossi bien & 42,2 & 0,000 \\
\hline Diminue les maladies chez l'enfant & 46,9 & 0,000 \\
\hline \multicolumn{3}{|l|}{ Autres avantages } \\
\hline Disponibilité permanente du lait & $\mathbf{9 , 6 1}$ & $\mathbf{0 , 0 5 2}$ \\
\hline maternel & 80,78 & $\mathbf{0 , 0 0 0}$ \\
\hline Economique & 9,61 & 0,052 \\
\hline Facile à utiliser & & \\
\hline
\end{tabular}




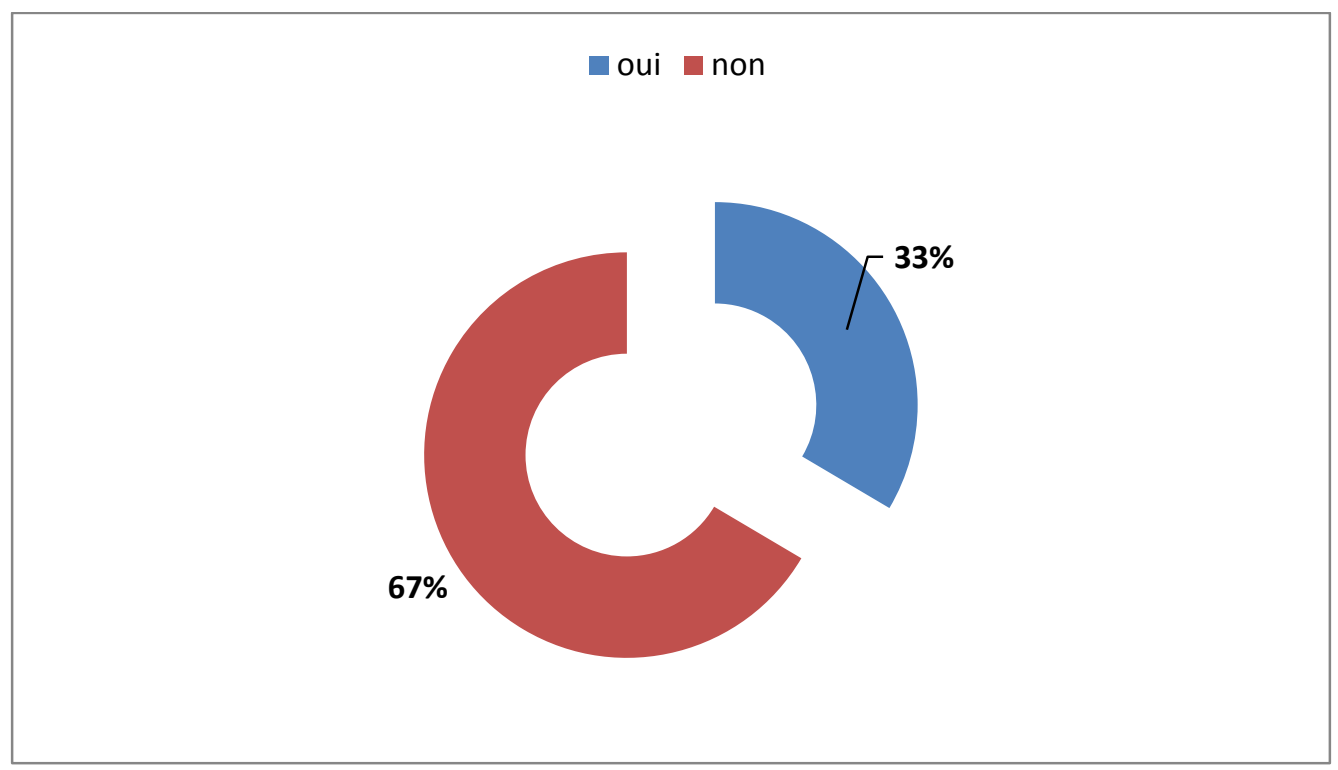

Figure 1 : Taux de l'allaitement exclusif de la zone d'étude.

Tableau 4: Facteurs sociodémographiques déterminant la pratique optimale de l'allaitement exclusif dans la zone d'étude.

\begin{tabular}{|c|c|c|c|}
\hline \multirow[b]{2}{*}{ Variables } & \multicolumn{2}{|c|}{ Pratique d'allaitement maternel exclusif } & \multirow[t]{2}{*}{ P-value } \\
\hline & Oui $(\%)$ & Non $(\%)$ & \\
\hline Tranche d'âge de la mère & & & $\mathbf{0 , 5 2 4}$ \\
\hline $15-24$ ans & 35,8 & 45,1 & \\
\hline $25-34$ ans & 50,8 & 43,6 & \\
\hline 35 ans et Plus & 13,4 & 11,3 & \\
\hline Niveau d'instruction & & & $\mathbf{0 , 8 2 1}$ \\
\hline Coranique & 25,4 & 24,8 & \\
\hline Analphabète & 17,9 & 21,1 & \\
\hline Primaire & 22,4 & 15,8 & \\
\hline Secondaire & 28,3 & 32,3 & \\
\hline Supérieur & 6 & 6 & \\
\hline Profession de la mère & & & $\mathbf{0 , 2 5 1}$ \\
\hline Elève/étudiante & 9 & 14,3 & \\
\hline Commerçante & 11,9 & 13 & \\
\hline Fonctionnaire & 6 & 1,5 & \\
\hline Ménagère & 62 & 70,7 & \\
\hline Couturière & 11,1 & 0,5 & \\
\hline Milieu de résidence & & & 0,953 \\
\hline Centre ville & 41,8 & 41,4 & \\
\hline Périphérie & 58,2 & 58,6 & \\
\hline Parité de la mère & & & 0,152 \\
\hline Primipare & 17,9 & 27,1 & \\
\hline Multipare & 82,1 & 72,9 & \\
\hline
\end{tabular}




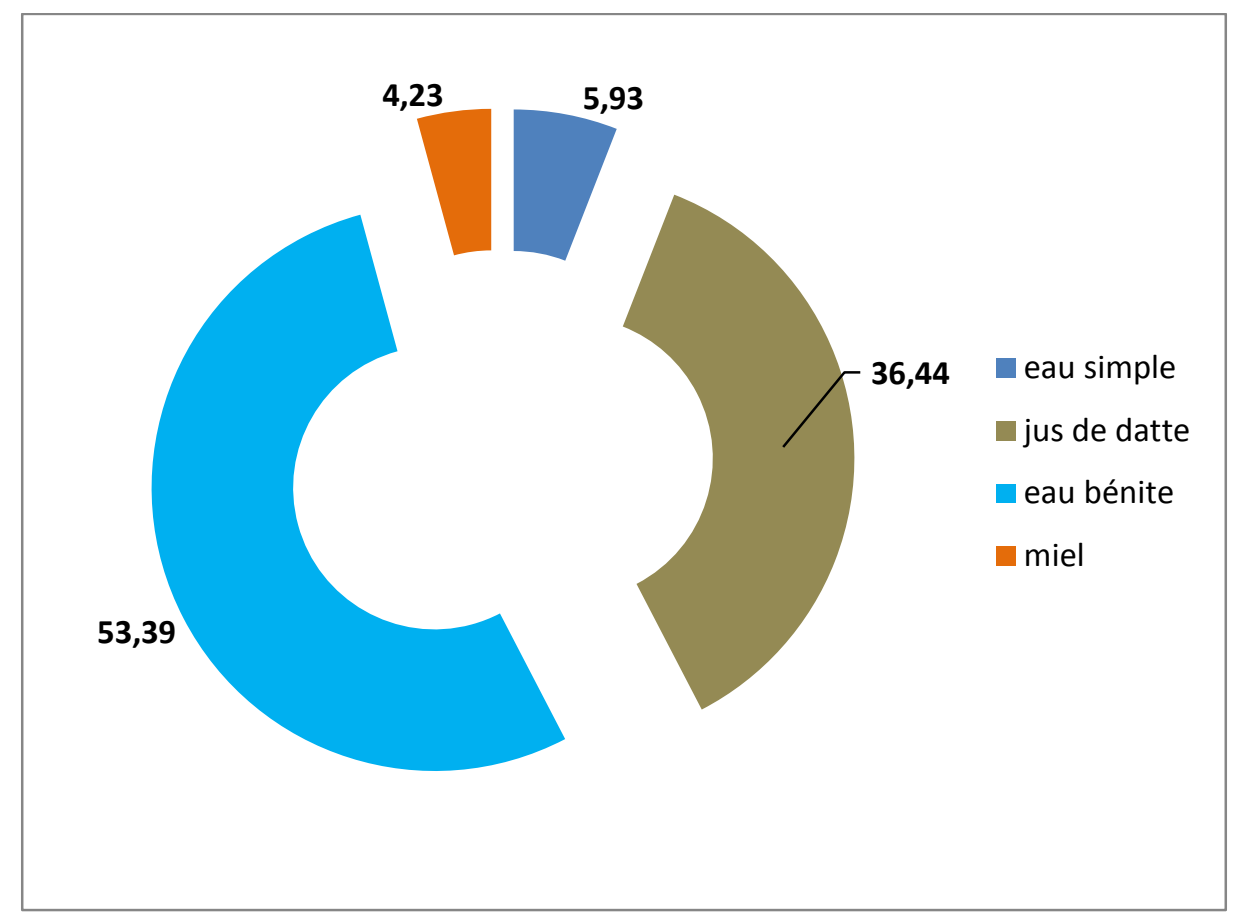

Figure 2 : Différents types d'aliments introduits dès la naissance chez l'enfant.

\section{DISCUSSION}

L'allaitement maternel exclusif ne concerne relativement que peu d'enfants encore à Niamey comme démontré par les résultats de cette étude. En effet, l'analyse a montré que seulement 33,5\% d'enfants ont bénéficié d'un allaitement maternel exclusif. Ce taux est supérieur à la moyenne nationale qui est de 23\% en 2012 selon INS (2012) mais reste inférieur au taux enregistré par INS en 2018 (56,6\%). Parallèlement, les résultats font ressortir une consommation très acceptable de colostrum par les enfants. En effet, 98\% d'enfants ont pris le colostrum bien que l'initiation à l'allaitement maternel a été étalée au-delà de 24 heures. Ce qui démontre l'abandon du rejet de colostrum qui est considéré dans certaines régions comme étant mauvais pour l'enfant. Les raisons avancées pour la prise de colostrum sont que le colostrum rend l'enfant fort et en bonne santé, et protège l'enfant contre les malades. Ainsi $48 \%$ d'enfants ont été initié à l'allaitement maternel dans l'heure qui suivit leur naissance,
$39 \%$ d'enfants mis au sein dans les 24 heures et enfin $11 \%$ enfants allaités pour la première fois qu'après 24 heures de naissance. Il convient de noter que ce taux de $48 \%$ pour cette étude est inférieur à la moyenne nationale de 2012 et de 2018 qui sont respectivement de $53 \%$ (INS, 2012) et $73,8 \%$ (INS, 2018) de mis au sein immédiat des enfants. Aussi, ce taux d'initiation à l'allaitement maternel est inférieur au taux enregistré par (Diadié, 2013). Par ailleurs, l'introduction précoce d'aliments a concerné $66,5 \%$ bien qu'elle ne soit pas recommandée officiellement chez le nourrisson. Cette pratique d'alimentation est en contradiction avec le niveau assez élevé des mères en matière de connaissance des contours de l'AME. En outre, la présente étude a révélé que la majorité des mères enquêtées ont entendu parler de l'AME très souvent au cours des consultations prénatales, infantiles et foraines. Un constat similaire a été aussi fait par UNICEF (2018) ayant observé un progrès dans la survie des enfants grâce à l'éducation nutritionnelle reçue par les mères. Par ailleurs, 
le test statistique a démontré qu'il n'existe aucune association significative avec les facteurs sociodémographiques des mères contrairement à l'étude au Laos réalisée par Kounnavong et al. (2013). Néanmoins, il a été relevé une association statistiquement significative entre l'AME et les variables suivantes : la connaissance de la durée de l'AME $(\mathrm{P}=0,000)$, la définition de l'AME $(\mathrm{P}=0,000)$, la connaissance de certains avantages de l'AME pour la mère: retarde la fertilité et renforce l'affection mère/enfant $(\mathrm{P}=0,005)$, la connaissance des avantages pour l'enfant : enfant grossit bien et en bonne santé $(\mathrm{P}=0,000)$, et enfin le lien avec le revenu des parents: économie sur l'achat du lait maternisé $(\mathrm{P}=0,000)$.

La connaissance des avantages sur l'AME est significativement associée à la pratique. En effet, les résultats de l'étude corroborent ceux de Maman (2012) et Youssoufa (2015) stipulant avec une $\mathrm{P}=0,004$ qu'une bonne connaissance des avantages de l'AME favoriserait sa pratique effective. Aussi, cette association significative enregistrée entre l'AME et les connaissances de mères en matière d'allaitement maternel a été prouvée par Sokol et al. (2007) et Tremeau (2017) qui proposèrent alors l'amélioration de l'alimentation du nourrisson et du jeune enfant à travers des conseils qualifiés sur l'AME à l'endroit des mères.

\section{Conclusion}

L'allaitement maternel exclusif se pratique encore au niveau de la commune urbaine de Niamey avec néanmoins un taux relativement bas de $33,5 \%$. Malgré, la volonté manifeste de certaines mères pour pratiquer efficacement l'AME, d'autres mères introduisent toutefois des aliments avant la première tétée chez près de $59 \%$ des enfants. Aussi, l'initiation précoce à l'allaitement demeure davantage puisqu'elle concerne environ $48 \%$ d'enfants de cette étude. Les résultats de l'analyse statistique ont montré une association significative entre la pratique de l'allaitement maternel exclusif et les facteurs tels que la connaissance de la durée et de la définition de l'AME, la connaissance de certains avantages de l'AME pour la mère (retarde la fertilité et renforce l'affection mère/enfant), la connaissance des avantages pour l'enfant (enfant grossit bien et en bonne santé), ainsi que le lien avec le revenu des parents(économie sur l'achat du lait non maternel).Ces résultats constituent des données de référence pour les voies d'amélioration de la prévalence de la malnutrition surtout chez le nourrisson de 0 à 6 mois au niveau de la communauté urbaine de Niamey.

\section{CONFLIT D'INTERETS}

Les auteurs déclarent qu'il n'y a pas de conflit d'intérêts pour cet article.

\section{REFERENCES}

Diadié OH. 2013. Pratiques d'alimentation du nourrisson et du jeune enfant au Niger : Etat des lieux, analyses technologique et nutritionnelle des aliments de complément et propositions d'amélioration. Nutrition humaine et technologie agroalimentaire, Thèse. Université Abdou Moumouni, Niamey, Niger. 146 pages.

INS. 2018. Nutrition et survie de l'enfant. Rapport technique, INS, Niger 75 pages.

INS. 2012. Nutrition et survie de l'enfant. Rapport technique. INS, Niger, 75 pages.

Kounnavong S, Pak-Gorstein S, Akkhavong K, Palaniappan U, Berdaga V, Conkle J, Gorstein J. 2013. Key determinants of optimal breastfeeding practices in Laos. Food and Nutrition Sciences, 4, 60-70. DOI:

http://dx.doi.org/10.4236/fns.2013.410A 010

Maman MS. 2012. Etude des pratiques d'alimentation des enfants âgés entre 0-6 mois issus des milieux défavorisés dans la commune urbaine de Tessaoua région de Maradi au Niger. Mémoire, Faculté des études supérieures et postdoctorales de l'université de Laval Québec.178 pages. 
MSP (Ministère de la Santé Publique). 2017. Stratégie national pour l'alimentation du nourrisson et du jeune enfant 2017-2025. Support technique. Niger, 90 pages.

Sokol E, Aguayo VM, Clark D, 2007. Protéger l'allaitement maternel en Afrique de l'Ouest et du Centre. 25 années d'application du Code International sur la Commercialisation des Substituts du Lait Maternel. Rapport technique UNICEF, Bureau Régional pour l'Afrique de l'Ouest et du Centre. 44 pages.

Tremeau V. 2017. L'insuffisance des investissements en faveur de l'allaitement nuit aux nourrissons et aux mères.
Rapport technique. UNICEF Niger. 22 pages.

UNICEF. 2013. L'allaitement maternel exclusif : une nécessité absolue. Rapport technique. 5 pages.

UNICEF. 2018. Rapport technique annuel. Niger. 74 pages.

Youssoufa DD. 2015. Étude des facteurs associés à l'allaitement maternel exclusif chez les mères d'enfants de $0-6$ mois à Beleko dans le District Sanitaire de Fana. Thèse de médecine et d'Odontostomatologie, Université des Sciences des Techniques et des technologies de Bamako Mali, 92 pages. 